\title{
Gene-Finding Strategy in Glaucoma
}

\author{
Mehrnaz Narooie-Nejad ${ }^{1,2,{ }^{*}}$ \\ ${ }^{1}$ Genetic of Non-Communicable Disease Research Center, Zahedan University of Medical Sciences, Zahedan, Iran \\ ${ }^{2}$ Genetics Department, Zahedan University of Medical Sciences, Zahedan, Iran \\ "Corresponding author: Genetic of Non-Communicable Disease Research Center, Zahedan University of Medical Sciences, Zahedan, Iran. Tel: +98-9153412628 , Email: \\ mehrnaznar@gmail.com
}

Received 2018 May 01; Revised 2018 August 05; Accepted 2018 August 07.

\begin{abstract}
Glaucoma is a group of complex eye disorders characterized by increased intraocular pressure, optic nerve damage, and ultimately, optic nerve cupping, ending in irreversible blindness. The age of onset and the anatomy of the anterior chamber indicate that glaucoma consists of the categories of primary open-angle glaucoma, primary congenital glaucoma, and primary angle closure glaucoma. To date, many loci have shown to be associated with different types of glaucoma; however, the underlying cause of the disease remains ambiguous. Two main approaches exist to find new loci and genes associated with the disease: linkage analysis, which is applicable for large pedigrees with multiple affected and healthy individuals, and a genome-wide association study for large cohorts of sporadic cases. Careful patient selection is the first step in these kinds of genetic research. All subjects selected should be examined to identify the known gene mutations for the specific type of glaucoma. Subjects with no mutations in known genes are possible subjects of a genome-wide association study or linkage analysis.
\end{abstract}

Keywords: Glaucoma, Genetic, Gene, Linkage Analysis, Association Study

\section{Glaucoma}

Glaucoma consists of a set of eye disorders characterized by increased intraocular pressure (IOP), optic nerve damage and ultimately, optic nerve cupping. Without early and effective treatment, glaucoma causes irreversible blindness (1-3). It is the second leading cause of blindness globally. Recent estimations put the number of glaucoma victims at about 70 million globally, with a $10 \%$ rate of blindness (4). Glaucoma is a complex disease with heterogeneous symptoms and an unidentified etiology (5). Glaucoma can be classified based on etiology (primary or secondary), the anatomy of the anterior chamber (open or closed angle), and age of onset (newborn, youth, or adult). The three main categories are primary open angle glaucoma (POAG), primary congenital glaucoma (PCG), and primary angle closure glaucoma (PACG). Another group with normal IOP, normal tension glaucoma (NTG), has been considered $(1,6)$.

There are many established risk factors for glaucoma, including high IOP, optic nerve cupping, age, race, family history, thin central corneal thickness (CCT), myopia, smoking, hypothyroidism, systolic hypertension, and diabetes (6-10). The role of ethnicity and family history in the development of glaucoma and its inheritability suggests the contribution of genetic factors to this disease (5). Inves- tigations have revealed the role of multiple loci in the establishment of glaucoma (2); however, the genetic basis for glaucoma has not been determined with certainty. Some individuals are affected by only one mutation and others by two or more mutations on different genes and show differences in the mode of inheritance (5). Finding out the individual endophenotypes alongside the genetic structure of the disease could be an important strategy for determining the etiology of glaucoma (5).

\section{Genetics of Glaucoma}

Glaucoma is a heterogeneous group of diseases with different patterns of symptoms and genetic bases. In firstdegree relatives of individuals affected by glaucoma, the risk of developing the disease is much greater. The etiology of glaucoma is unknown; thus, there are no direct clues to find the genetic basis of the disease. The role of environmental factors in glaucoma, the phenotypes of those affected, the diversity of effective treatment approaches in patients, and the diversity of family histories add to the complexity of the disease. The detection of several loci for glaucoma, different Mendelian inheritance patterns, and incomplete penetrance of the disease are also the other factors that add to the complexity of glaucoma. As in other re- 
ports on digenic inheritance in complex diseases, this has been reported in glaucoma. Each class of glaucoma has been identified using different causative genes and genetic patterns.

\section{Primary Open Angle Glaucoma}

POAG (OMIM137760) is the major form of glaucoma (3). It currently affects more than 70 million people worldwide (11), estimated to affect about 111.8 million by 2040 (10). POAG is classified as juvenile open-angle glaucoma (JOAG), with an onset primarily in young adults above three years of age and adult-onset POAG, with an onset of over 40 years of age (12). The disease also can be divided into two types according to the IOP: high-tension glaucoma (HTG) and normal-tension glaucoma (NTG) (13).

Although glaucoma is characterized as complex, in most cases, POAG is inherited through the autosomal dominant mode of inheritance, which suggests the involvement of single-gene mutations in the pathogenesis of the disease $(8,10,14)$. Linkage analysis (LA) and association studies of large families have mapped several susceptible loci for POAG (GLC1A-P) (14-16). Among these, only three genes are known to contribute to POAG: MYOC (myocilin at GLC1A; OMIM 601652) (17), OPTN (optineurin at GLC1E and OMIM 602432) (18), and WDR36 (WD repeat domain 36 at GLC1G; OMIM 609669) (19), causing less than $10 \%$ of the disease (20).

The mechanism of myocilin function in relation to the disease is unclear. Disease-associated mutations of the MYOC gene are primarily responsible for JOAG and early onset of the adult form of the disease. These patients suffer from high IOP. Besides high IOP, family history is considered a risk factor for POAG, with a relative risk of 9.2 in populations with positive family histories (21). Finding out the relative risk could be very valuable in predicting the status of the disease in a population.

Any approach to detect early signs of glaucoma is crucial to prevent irreversible damage by the disease. There is evidence that the disease-causing mutation of the MYOC gene causes misfolding of the MYOC protein, which leads to the accumulation of the protein in the intracellular environment. The transportation of the protein faces many obstacles (20)

Another gene proposed for POAG is WDR36. It is believed to be associated/or the causative agent of the disease in many studies (13), but conflicts exist about the role of this gene in the pathogenesis of POAG. It recently has been suggested that WDR36, although is a susceptibility factor for POAG, is not primarily responsible for it.

In a study of families with NTG, the association of the OPTN gene with POAG was discovered. It has been sug- gested in some studies that this gene is involved in nerve protection and mutations that cause a disorder in the protein produced by this gene are involved in optic nerve damage in glaucoma (22). Mutations in OPTN are responsible for less than $1.5 \%$ of all types of glaucoma; however, the role of OPTN mutation in the development of POAG requires further investigation. The TBK1 (TANK-binding kinase 1) (23), NTF4 (neurotrophin-4) $(24,25)$ and GSTM1 (glutathione S-transferase mu 1) $(26,27)$ genes are involved in the pathogenesis of POAG but have not been studied in many populations.

Mutations in all of these genes account for less than $10 \%$ of the POAG patients in several populations $(15,26)$. A study in Iran found that $17.4 \%$ of the JOAG patients carried heterozygote MYOC mutations (28), but these mutations were found in less than $1 \%$ of the POAG patients in southeastern Iran (data not published). The role of MYOC mutations in PCG has observed only in a few studies (23, 24, 29) in association with genes such as common variants of the CAV1/CAV2 gene region (20,30), CDKN2BAS, TMCO1, and SIX1/SIX6 genes (30).

\section{Primary Congenital Glaucoma}

Primary congenital glaucoma (OMIM 231300; infantile glaucoma) is a rare form of glaucoma that affects children under three years of age (31-33), mostly in the autosomal recessive mode of inheritance in familial cases (30). The disease has been observed as being sporadic in many cases. PCG is a developmental disorder of the trabecular meshwork and anterior chamber angle, which draws back the aqueous outflow and creates a significant increase in intraocular pressure at birth and early childhood. Other symptoms of the disease are enlargement of the eyeball (buphthalmos), edema of the cornea, enlarged diameter of the cornea (megalocornea), opacification, fracture of the Descemet membrane, iris atrophy, and anomalies of the anterior chamber. These ultimately result in optic nerve damage and loss of vision (34). Blepharospasm, photophobia, and epiphora are the primary symptoms of the disease. It has been reported that the incidence of the disease shows geographic variability, with higher ratios in populations having a higher rate of consanguineous marriages (32).

PCG, like other forms of glaucoma, has heterogeneity in its genetic basis. Of the four main loci found by linkage studies for PCG $(1,2,26,35,36)$, only two contribute to PCG, including CYP1B1 (cytochrome P450 1B1) (35) and LTBP2 (latent transforming growth factor binding protein 2) $(34,37)$. CYP1B1 (NM_000104.3) is located on chromosome 2p22-p21 in the GLC3A locus (OMIM 231300) and contains three exons and two introns. The protein encoded by 
this gene is 534 amino acids in length with a putative open reading frame of 1632 base pairs (34). It has been shown that the coding sequence of CYP1B1 contains exons 2 and 3. In fact, exon 1 is noncoding (38). It has been suggested that the gene product may involve in the metabolic pathway of signaling molecules, which is critical to the development of the iridocorneal angle, but this theory has not been confirmed $(33,39)$.

The gene is highly expressed in ocular tissues and is known as the predominant cause of PCG in different populations $(4,34)$. Reports indicate that several CYP1B1 mutations in different societies have been associated with different phenotypes of the disease (mild, moderate, and severe). In the first study conducted in Iran, CYP1B1 mutations were responsible for PCG in 70\% (40) and for JOAG in $17.4 \%$ (28) of patients but were not responsible for POAG and JOAG in southeastern Iran (41). CYP1B1 mutations show incomplete penetrance in the Iranian population (32). A few studies have revealed the role of CYP1B1 mutations in JOAG $(28,42)$, suggesting a relationship between the CYP1B1 and MYOC proteins (26) although the results in different populations varied significantly.

Besides the genetic basis of the disease, epigenetics and environmental factors have played a significant role in the development of the disease (39). LTBP2 (NM_000428.2) is located on chromosome $14 \mathrm{q} 24$ in the GLC3D locus. It is expressed in the trabecular meshwork of the eye and involved in ciliary zonule microfibrils $(2,15)$. Null mutations in this gene have been reported in PCG patients from Iran, Pakistan, and Romania, but not in other populations. Many studies have revealed the association of LTBP2 mutations with secondary glaucoma, but the precise role of this gene in PCG is not clear (33). Mutations in this gene may be involved in PACG (27).

In the two remaining loci associated with PCG, GLC3B (gene ID: 2728, OMIM \#600975;1p36.2-p36.1 (26) and GLC3C (gene ID:399565, OMIM \#613085; 14q24.2-q24.3)(2), no gene has been found. There is evidence that other genes may be involved in the onset of PCG. Reports from India have suggested a role for the MYOC gene(OMIM \#601652) $(13,43)$ and FOXC1 gene (OMIM \#601090) (44) in a few patients affected by PCG.

\section{Primary Angle Closure Glaucoma}

The presence of mechanical obstructions in the trabecular meshwork causes angle closure in the anterior chamber of the eye (45). PACG, as the second major form of glaucoma, affects $10 \%$ of the adults over 60 years of age with a greater frequency in men and a complex mode of inheritance. As for other forms of glaucoma, there is evidence that one or more genes contribute to PACG (6). Although several genetic loci have been introduced as genetic factors of PACG, the only gene known as a causative agent of PACG is NNO1 (nanophthalmos 1) (36). Some variants have a positive association with PACG but do not cause the disease (6, 16, 46). Associations with PLEKHA7 (rs11024102) and COL11A1 (rs3753841) have been reported in some populations from genome-wide association studies (GWASs) (31, 45). These associations have been confirmed by other researchers $(47,48)$. It is believed these two genes are related to the acute form of PACG (48). Through GWASs, several polymorphisms have been revealed on the candidate genes associated with PACG and they can be exemplified by single nucleotide polymorphisms (SNPs) on eNOS (endothelial nitric oxide synthase) (49), ABCC5 (ATP binding cassette subfamily C member 5) (50), MFRP (membrane type frizzledrelated protein), HSP70 (heat-shock protein 70) (49), and MMP9 (matrix metalloproteinase-9) genes (48). Because the etiology of PACG remains unknown, there is a need for more studies.

Many other genes are associated with several kinds of glaucoma and associated diseases. These include TBK1 and OPTN for NTG (51), PITX2, PAX6, and FOXC1 for developmental glaucoma (Axenfeld-Rieger syndrome, aniridia) $(16,46)$ and LOXL1 for exfoliation syndrome (34).

\section{The Path from Gene to Disease}

Finding out the role of genetic factors in the development of a complex multifactorial disease helps to find the pathogenic pathway of the disease (6). In addition, if genetic factors are considered as biomarkers, they could be used for disease diagnosis and/or prognosis (6). LA and GWASs are the main approaches to find the correlation between a candidate gene and a common or complex disorder (7). Using extensive knowledge about genetic markers and automatic genotyping techniques has it possible to provide genetic and physical maps (52). In both LA and GWAS methods, genetic variants have been investigated in a population to find its association with a phenotype (or trait).

A genetic variant is in a linkage disequilibrium (LD) with a trait where it has a functional effect on it or it is linked to the disease-causing gene $(2,4)$. In such approaches, known DNA markers (scattered along the genome), like SNPs and microsatellites, have been evaluated in many individuals in different generations in family cases or in many affected individuals in sporadic cases. Wherever the markers are a part of a functional gene or in an LD with it, they will co-segregate with the trait in the studied subjects. It means that all affected individuals in these evaluations show a unique genotype pattern 
of markers in a specific region of the genome (a locus), but healthy controls do not.

The basic theory is that all affected individuals in a pedigree have received the disease-causing allele or alleles (like markers that are in an LD with that gene) from a common ancestor. Loci with such markers are the first estimates for the loci-containing genes associated with the disease. Parametric or non-parametric LD screens the entire genome to localize the genes responsible for a trait (24). It should be conducted for large pedigrees with a number of affected and non-affected individuals. These pedigrees are valuable but are uncommon and inaccessible in most cases (53). Incomplete penetrance, population stratification, locus heterogeneity, and phenocopy can distort the results.

Because of the limits to linkage studies to find large pedigrees, most studies have shifted toward GWAS. The first GWAS was published in 2002 and found a candidate locus on chromosome 6p21 associated with susceptibility to myocardial infarction (54). This approach scans the entire genome for genetic variants (or polymorphisms) between affected individuals and healthy controls. A positive association is considered when the frequency of specific genetic variants is greater in the affected individuals than in the control group. This means that there is an association between this region of the genome (which contains the disease-causing gene) and the disease.

The accuracy of GWAS has improved with the use of DNA chips having more than a million SNPs $(4,6)$. As opposed to linkage studies in which there is a need for large pedigrees, GWASs are conducted mostly in a large cohort. The two main types of cohorts are the independent population (case-control study) in sporadic cases and the familial cases, even those with small pedigrees (7). In all the approaches, GWAS requires a large number of samples, which increases costs $(2,33)$.

\section{Patient Selection and Analysis of Genes to Find a Strategy for Glaucoma}

There should be a detailed plan for all types of GWAS or LA. Careful patient selection is the first step in such genetic research; thus, there is a need for definition and classification of glaucomatous patients. As each type of glaucoma has a specific genetic basis, all patients should be clinically examined to determine the exact type of glaucoma. Patients with any other type of disease should be excluded from the study. Next, the mode of inheritance should be ascertained. This is most difficult for sporadic cases. Large pedigrees with multiple affected and healthy individuals are suitable for LA. In parametric LA, susceptibility factors should be determined. These include gene frequency, penetrance, and mode of inheritance. Because having access to such information is not always possible, non-parametric linkage methods are used instead. In this regard, affected individuals and their relatives are the subjects of LA to find the chromosomal area that is common to affected subjects. The best example of this approach is the affected sib-pair analysis. In all the pedigrees analyzed by these methods, informative meiosis is a necessity $(39,53)$.

As large pedigrees are not commonly available, most strategies focus on sporadic cases. In sporadic cases that are suitable for association studies, there is a need for a large cohort of affected and healthy samples to conduct a case-control study. In the LA method, microsatellites and SNPs are both suitable as markers while GWAS applies dense SNP arrays. This is because GWAS is conducted on unrelated people of a population who supposedly have a common ancestor with a susceptibility factor $(15,23,33)$.

All patients selected should be examined to identify known gene mutations related to the type of glaucoma. After screening, remaining patients with no mutations in known genes will become the subjects of GWAS or LA. The CYP1B1 and MYOC genes should be screened first to find the genetic basis of glaucoma in each population.

Both LA and GWAS are difficult and error-prone and should be performed with high precision. If the samples are informative, the disease-associated locus should be identified using these approaches. Each locus contains many genes, the amount (or locus size) of which depends on how informative are the pedigrees or is determined by chance. All the genes should be evaluated by DNA sequencing, which is expensive and time-consuming. Once diseaserelated genes are found, the results should be verified. In other words, mutations in the candidate gene should be present in all patients, but not in the healthy individuals. To finalize the procedure, the function of the candidate gene should be analyzed. The expression of the candidate gene is evaluated in the related tissues and it should be in conformity with the glaucoma phenotype (37). At this step, the function of the mutated gene should be monitored, either in silico (55) or in laboratory animals (33). The pathway from the disease to the gene is a multi-step procedure that must be undertaken with great care.

\section{References}

1. Lim SH, Tran-Viet KN, Yanovitch TL, Freedman SF, Klemm T, Call W, et al. CYP1B1, MYOC, and LTBP2 mutations in primary congenital glaucoma patients in the United States. Am J Ophthalmol. 2013;155(3):508517 e5. doi: 10.1016/j.ajo.2012.09.012. [PubMed: 23218701]. [PubMed Central: PMC3736560].

2. Schlotzer-Schrehardt U, Zenkel M, Kuchle M, Sakai LY, Naumann GO. Role of transforming growth factor-beta1 and its latent form binding protein in pseudoexfoliation syndrome. Exp Eye Res. 2001;73(6):76580. doi:10.1006/exer.2001.1084. [PubMed: 11846508]. 
3. Nakano M, Ikeda Y, Taniguchi T, Yagi T, Fuwa M, Omi N, et al. Three susceptible loci associated with primary open-angle glaucoma identified by genome-wide association study in a Japanese population. Proc Natl Acad Sci U S A. 2009;106(31):12838-42. doi: 10.1073/pnas.0906397106. [PubMed: 19625618]. [PubMed Central: PMC2722348].

4. Teixeira LB, Zhao Y, Dubielzig RR, Sorenson CM, Sheibani N. Ultrastructural abnormalities of the trabecular meshwork extracellular matrix in Cyp1b1-deficient mice. Vet Pathol. 2015;52(2):397-403. doi: 10.1177/0300985814535613. [PubMed: 24879660]. [PubMed Central: PMC4285769].

5. Duggal P, Ibay G, Klein AP. Current gene discovery strategies for ocular conditions. Invest Ophthalmol Vis Sci. 2011;52(10):7761-70. doi: 10.1167/iovs.10-6989. [PubMed: 21960645]. [PubMed Central: PMC3183987].

6. Ahram DF, Alward WL, Kuehn MH. The genetic mechanisms of primary angle closure glaucoma. Eye (Lond). 2015;29(10):1251-9. doi: 10.1038/eye.2015.124. [PubMed: 26206529]. [PubMed Central: PMC4815686].

7. Zhou M, Wang W, Huang W, Zhang X. Diabetes mellitus as a risk factor for open-angle glaucoma: a systematic review and meta-analysis. PLoS One. 2014;9(8). e102972. doi: 10.1371/journal.pone.0102972. [PubMed: 25137059]. [PubMed Central: PMC4138056].

8. Garcia-Albornoz M, Nielsen J. Finding directionality and genedisease predictions in disease associations. BMC Syst Biol. 2015;9:35. doi: 10.1186/s12918-015-0184-9. [PubMed: 26168918]. [PubMed Central: PMC4501277].

9. Charlesworth J, Kramer PL, Dyer T, Diego V, Samples JR, Craig JE, et al. The path to open-angle glaucoma gene discovery: endophenotypic status of intraocular pressure, cup-to-disc ratio, and central corneal thickness. Invest Ophthalmol Vis Sci. 2010;51(7):350914. doi: 10.1167/iovs.09-4786. [PubMed: 20237253]. [PubMed Central: PMC2904007].

10. Abu-Amero K, Kondkar AA, Chalam KV. An updated review on the genetics of primary open angle glaucoma. Int $\mathrm{J} \mathrm{Mol}$ Sci. 2015;16(12):28886-911. doi: 10.3390/ijms161226135. [PubMed: 26690118]. [PubMed Central: PMC4691082].

11. Pleet A, Sulewski M, Salowe RJ, Fertig R, Salinas J, Rhodes A, et al. Risk factors associated with progression to blindness from primary open-angle glaucoma in an African-American population. Ophthalmic Epidemiol. 2016;23(4):248-56. doi: 10.1080/09286586.2016.1193207. [PubMed: 27348239]. [PubMed Central: PMC4939437].

12. Wang R, Wiggs JL. Common and rare genetic risk factors for glaucoma. Cold Spring Harb Perspect Med. 2014;4(12). a017244. doi: 10.1101/cshperspect.a017244. [PubMed: 25237143]. [PubMed Central: PMC4292091].

13. Kaur K, Reddy AB, Mukhopadhyay A, Mandal AK, Hasnain SE, Ray $\mathrm{K}$, et al. Myocilin gene implicated in primary congenital glaucoma. Clin Genet. 2005;67(4):335-40. doi: 10.1111/j.1399-0004.2005.00411.x. [PubMed: 15733270].

14. Scheetz TE, Fingert JH, Wang K, Kuehn MH, Knudtson KL, Alward $\mathrm{WL}$, et al. A genome-wide association study for primary open angle glaucoma and macular degeneration reveals novel Loci. PLoS One. 2013;8(3). e58657. doi: 10.1371/journal.pone.0058657. [PubMed: 23536807]. [PubMed Central: PMC3594156].

15. Inoue T, Ohbayashi T, Fujikawa Y, Yoshida H, Akama TO, Noda K, et al. Latent TGF-beta binding protein-2 is essential for the development of ciliary zonule microfibrils. Hum Mol Genet. 2014;23(21):567282. doi: 10.1093/hmg/ddu283. [PubMed: 24908666]. [PubMed Central: PMC4189902].

16. Shastry BS. Genetic susceptibility to primary angle closure glaucoma (PACG). Discov Med. 2013;15(80):17-22. [PubMed: 23375010].

17. Stone EM, Fingert JH, Alward WL, Nguyen TD, Polansky JR, Sunden SL, et al. Identification of a gene that causes primary open angle glaucoma. Science. 1997;275(5300):668-70. [PubMed: 9005853].

18. Rezaie T, Child A, Hitchings R, Brice G, Miller L, Coca-Prados M, et al. Adult-onset primary open-angle glaucoma caused by mutations in optineurin. Science. 2002;295(5557):1077-9. doi: 10.1126/science.1066901. [PubMed: 11834836].

19. Monemi S, Spaeth G, DaSilva A, Popinchalk S, Ilitchev E, Liebmann J, et al. Identification of a novel adult-onset primary open-angle glaucoma (POAG) gene on 5q22.1. Hum Mol Genet. 2005;14(6):725-33. doi: 10.1093/hmg/ddi068. [PubMed: 15677485].

20. Weinreb RN, Aung T, Medeiros FA. The pathophysiology and treatment of glaucoma: a review. JAMA. 2014;311(18):1901-11. doi: 10.1001/jama.2014.3192. [PubMed: 24825645]. [PubMed Central: PMC4523637].

21. Vegini F, Figueiroa Filho N, Lenci RF, Garcia Neto D, Susanna Junior R. Prevalence of open angle glaucoma in accompanying first degree relatives of patients with glaucoma. Clinics (Sao Paulo). 2008;63(3):32932. [PubMed: 18568241]. [PubMed Central: PMC2664248].

22. Fingert JH. Primary open-angle glaucoma genes. Eye (Lond). 2011;25(5):587-95. doi: 10.1038/eye.2011.97. [PubMed: 21562585]. [PubMed Central: PMC3171270].

23. Tanwar M, Kumar M, Dada T, Sihota R, Dada R. MYOC and FOXC1 gene analysis in primary congenital glaucoma. Mol Vis. 2010;16:1996-2006. [PubMed: 21031026]. [PubMed Central: PMC2956699].

24. Hilal L, Boutayeb S, Serrou A, Refass-Buret L, Shisseh H, Bencherifa F, et al. Screening of CYP1B1 and MYOC in Moroccan families with primary congenital glaucoma: three novel mutations in CYP1B1. Mol Vis. 2010;16:1215-26. [PubMed: 20664688]. [PubMed Central: PMC2901188].

25. Pasutto F, Matsumoto T, Mardin CY, Sticht H, Brandstatter JH, Michels-Rautenstrauss $\mathrm{K}$, et al. Heterozygous NTF4 mutations impairing neurotrophin-4 signaling in patients with primary open-angle glaucoma. Am J Hum Genet. 2009;85(4):447-56. doi: 10.1016/j.ajhg.2009.08.016. [PubMed: 19765683]. [PubMed Central: PMC2756554].

26. Dong S, Yang J, Yu W, Kota P, Xia X, Xu H. No association of genetic polymorphisms in CYP1B1 with primary open-angle glaucoma: a metaand gene-based analysis. Mol Vis. 2012;18:786-96. [PubMed: 22509109]. [PubMed Central: PMC3324367].

27. Safari I, Akbarian S, Yazdani S, Elahi E. A possible role for LTBP2 in the etiology of primary angle closure glaucoma.J Ophthalmic Vis Res. 2015;10(2):123-9. doi: 10.4103/2008-322X.163783. [PubMed: 26425313]. [PubMed Central: PMC4568608].

28. Bayat B, Yazdani S, Alavi A, Chiani M, Chitsazian F, Tusi BK, et al. Contributions of MYOC and CYP1B1 mutations to JOAG. Mol Vis. 2008;14:50817. [PubMed: 18385784]. [PubMed Central: PMC2268862].

29. Elahi E, Narooie-Nejhad M, Suri F, Yazdani S. Myocilin mutations are not a major cause of primary congenital glaucoma in Iranian patients. J Ophthalmic Vis Res. 2010;5(2):101-4. [PubMed: 22737338]. [PubMed Central: PMC3380681].

30. Kelberman D, Islam L, Lakowski J, Bacchelli C, Chanudet E, Lescai F, et al. Mutation of SALL2 causes recessive ocular coloboma in humans and mice. Hum Mol Genet. 2014;23(10):2511-26. doi: 10.1093/hmg/ddt643. [PubMed: 24412933]. [PubMed Central: PMC3990155].

31. Vink JM, Boomsma DI. Gene finding strategies. Biol Psychol. 2002;61(12):53-71. doi: 10.1016/s0301-0511(02)00052-2.

32. Achary MS, Reddy AB, Chakrabarti S, Panicker SG, Mandal AK, Ahmed $\mathrm{N}$, et al. Disease-causing mutations in proteins: structural analysis of the CYP1B1 mutations causing primary congenital glaucoma in humans. Biophys J. 2006;91(12):4329-39. doi: 10.1529/biophysj.106.085498. [PubMed: 16963504]. [PubMed Central: PMC1779944].

33. Moore DB, Tomkins O, Ben-Zion I. A review of primary congenital glaucoma in the developing world. Surv Ophthalmol.2013;58(3):278-85. doi: 10.1016/j.survophthal.2012.11.003. [PubMed: 23465868].

34. Li N, Zhou Y, Du L, Wei M, Chen X. Overview of Cytochrome P450 $1 \mathrm{~B} 1$ gene mutations in patients with primary congenital glaucoma. Exp Eye Res. 2011;93(5):572-9. doi: 10.1016/j.exer.2011.07.009. [PubMed: 
21854771].

35. Sarfarazi M, Akarsu AN, Hossain A, Turacli ME, Aktan SG, BarsoumHomsy M, et al. Assignment of a locus (GLC3A) for primary congenital glaucoma (Buphthalmos) to 2p21 and evidence for genetic heterogeneity. Genomics. 1995;30(2):171-7. [PubMed: 8586416].

36. Narooie-Nejad M, Chitsazian F, Khoramian Tusi B, Mousavi F, Houshmand M, Rohani MR, et al. Genotyping results of Iranian PCG families suggests one or more PCG locus other than GCL3A, GCL3B, and GCL3C exist. Mol Vis. 2009;15:2155-61. [PubMed: 19898634]. [PubMed Central: PMC2773737].

37. Narooie-Nejad M, Paylakhi SH, Shojaee S, Fazlali Z, Rezaei Kanavi $\mathrm{M}$, Nilforushan $\mathrm{N}$, et al. Loss of function mutations in the gene encoding latent transforming growth factor beta binding protein 2, LTBP2, cause primary congenital glaucoma. Hum Mol Genet. 2009;18(20):3969-77. doi:10.1093/hmg/ddp338. [PubMed: 19656777].

38. Tang YM, Wo YY, Stewart J, Hawkins AL, Griffin CA, Sutter TR, et al. Isolation and characterization of the human cytochrome P450 CYP1B1 gene. J Biol Chem. 1996;271(45):28324-30. [PubMed: 8910454].

39. Shastry BS. Emerging concept of genetic and epigenetic contribution to the manifestation of glaucoma. Glaucoma-Basic and Clinical Aspects. IntechOpen; 2013. doi: 10.5772/52279.

40. Chitsazian F, Tusi BK, Elahi E, Saroei HA, Sanati MH, Yazdani S, et al. CYP1B1 mutation profile of Iranian primary congenital glaucoma patients and associated haplotypes. J Mol Diagn. 2007;9(3):382-93. doi: 10.2353/jmoldx.2007.060157. [PubMed: 17591938]. [PubMed Central: PMC1899412].

41. Narooie-Nejad M, Rigi E, Torkamanzehi A, Rohani MR. A study of CYP1B1 gene prevalent mutations in patients with primary openangle glaucoma in Sistan and Baluchestan province, Iran. Gene Cell Tissue. 2016;3(2). doi: 10.17795/gct-33668.

42. Vincent AL, Billingsley G, Buys Y, Levin AV, Priston M, Trope G, et al. Digenic inheritance of early-onset glaucoma: CYP1B1, a potential modifier gene. Am J Hum Genet. 2002;70(2):448-60. doi: 10.1086/338709. [PubMed: 11774072]. [PubMed Central: PMC384919].

43. Chakrabarti S, Kaur K, Komatireddy S, Acharya M, Devi KR, Mukhopadhyay A, et al. Gln48His is the prevalent myocilin mutation in primary open angle and primary congenital glaucoma phenotypes in India. Mol Vis. 2005;11:111-3. [PubMed: 15723004].

44. Chakrabarti S, Kaur K, Rao KN, Mandal AK, Kaur I, Parikh RS, et al. The transcription factor gene FOXC1 exhibits a limited role in primary congenital glaucoma. Invest Ophthalmol Vis Sci. 2009;50(1):75-83. doi: 10.1167/iovs.08-2253. [PubMed: 18708620].
45. Sun X, Dai Y, Chen Y, Yu DY, Cringle SJ, Chen J, et al. Primary angle closure glaucoma: What we know and what we don't know. Prog Retin Eye Res. 2017;57:26-45. doi: 10.1016/j.preteyeres.2016.12.003. [PubMed: 28039061].

46. Shi H, Zhu R, Hu N, Shi J, Zhang J, Jiang L, et al. An extensive replication study on three new susceptibility Loci of primary angle closure glaucoma in han chinese: jiangsu eye study. J Ophthalmol. 2013;2013:641596. doi: 10.1155/2013/641596. [PubMed: 24282630]. [PubMed Central: PMC3824414].

47. Awadalla MS, Thapa SS, Hewitt AW, Burdon KP, Craig JE. Association of genetic variants with primary angle closure glaucoma in two different populations. PLoS One. 2013;8(6). e67903. doi 10.1371/journal.pone.0067903. [PubMed: 23840785]. [PubMed Central: PMC3695871].

48. Chen Y, Chen X, Wang L, Hughes G, Qian S, Sun X. Extended association study of PLEKHA7 and COL11A1 with primary angle closure glaucoma in a Han Chinese population. Invest Ophthalmol Vis Sci. 2014;55(6):3797-802. doi: 10.1167/iovs.14-14370. [PubMed: 24854855].

49. Ayub H, Khan MI, Micheal S, Akhtar F, Ajmal M, Shafique S, et al. Association of eNOS and HSP70 gene polymorphisms with glaucoma in Pakistani cohorts. Mol Vis. 2010;16:18-25. [PubMed: 20069064]. [PubMed Central: PMC2805420].

50. Nongpiur ME, Sakata LM, Friedman DS, He M, Chan YH, Lavanya R et al. Novel association of smaller anterior chamber width with angle closure in Singaporeans. Ophthalmology. 2010;117(10):1967-73. doi: 10.1016/j.ophtha.2010.02.007. [PubMed: 20541809].

51. Schaffer AA. Digenic inheritance in medical genetics. J Med Genet 2013;50(10):641-52. doi: 10.1136/jmedgenet-2013-101713. [PubMed: 23785127]. [PubMed Central: PMC3778050].

52. Pulst SM. Genetic linkage analysis. Arch Neurol. 1999;56(6):667-72. [PubMed: 10369304].

53. Stoilov I, Sarfarazi M. The third genetic locus (GLC3C) for primary congenital glaucoma (PCG) maps to chromosome 14q24.3. Investig Ophthalmol Vis Sci. 2002;43(13).

54. Ozaki K, Ohnishi Y, Iida A, Sekine A, Yamada R, Tsunoda T, et al. Functional SNPs in the lymphotoxin-alpha gene that are associated with susceptibility to myocardial infarction. Nat Genet. 2002;32(4):650-4. doi: 10.1038/ng1047. [PubMed: 12426569].

55. Patnala R, Clements J, Batra J. Candidate gene association studies: a comprehensive guide to useful in silico tools. BMC Genet. 2013;14:39. doi: 10.1186/1471-2156-14-39. [PubMed: 23656885]. [PubMed Central: PMC3655892]. 